\title{
INTERMITÊNCIA DO SISTEMA DE RESFRIAMENTO ADIABÁTICO EVAPORATIVO POR ASPERSÃO EM INSTALAÇÃO PARA VACAS EM LACTAÇÃO ${ }^{1}$
}

\section{SORAIA V. MATARAZZO ${ }^{2}$, IRAN J. O. DA SILVA ${ }^{3}$, MAURICIO PERISSINOTTO ${ }^{4}$, SÉRGIO A. DE A. FERNANDES ${ }^{5}$}

\begin{abstract}
RESUMO: Teve-se o objetivo de avaliar o período de intermitência de acionamento do sistema de resfriamento adiabático evaporativo (SRAE) por aspersão na linha de alimentação, em instalação do tipo freestall e sua influência no conforto térmico de vacas em lactação. Foram utilizadas 12 vacas multíparas com produção média de $30 \mathrm{~kg}$ de leite por dia. Os tratamentos corresponderam às intermitências de 12; 14 e 16 min, e os dados referentes à temperatura do bulbo seco (TBS), umidade relativa do ar (UR) e temperatura de globo negro (TGN), no interior da instalação e no ambiente externo, foram mensurados ao longo das $24 \mathrm{~h}$ durante todo o período experimental. A freqüência respiratória (FR), temperatura retal (TR) e temperatura do pelame (TP) foram medidas às $13 ; 14 ; 15$ e $16 \mathrm{~h}$ em todos os animais. As variáveis meteorológicas TBS $\left(31,5^{\circ} \mathrm{C}\right)$, TGN $\left(32,5^{\circ} \mathrm{C}\right)$ e os índices de conforto térmico ITU $(79,0)$ e ITGU $(81,0)$ foram maiores na intermitência de 12 min quando comparados aos demais tratamentos. A UR mostrou-se mais elevada no tratamento de 14 min $(60,3 \%)$ em relação à intermitência de 12 min $(51,0 \%)$. Verificou-se que as vacas em lactação expostas à intermitência de $12 \mathrm{~min}$ apresentaram maior valor de TR $\left(39,1^{\circ} \mathrm{C}\right)$ e $\mathrm{FR}\left(76,0\right.$ mov $\left.\mathrm{min}^{-1}\right)$ quando comparado aos tratamentos de 14 e $16 \mathrm{~min}$. As intermitências de 14 e 16 min proporcionaram condições ambientais semelhantes e menores valores de TR, FR e TP, mostrando-se eficientes na redução do estresse térmico de vacas em lactação. O intervalo de 16 min empregou menor quantidade de água, podendo ser adotado a fim de evitar desperdícios desnecessários pelo sistema de resfriamento adiabático evaporativo.
\end{abstract}

PALAVRAS-CHAVE: ambiência, vacas em lactação, conforto térmico.

\section{SPRINKLING FREQUENCY OF ADIABATIC EVAPORATIVE COOLING SYSTEM IN DAIRY COW HOUSING}

ABSTRACT: This work aimed to evaluate the effect of sprinkling frequency of adiabatic evaporative cooling system within feed line of a freestall in relation to temperature and humidity index (THI),black globe temperature index (BGHI) and physiological responses in dairy cows. There were used twelve multiparous dairy cows with medium milk production of $30 \mathrm{~kg}$ per day. The treatments correspond to sprinkling pulses of $12 ; 14$ and 16 min sprinkler system. Dry bulb temperature (DBT), air relative humidity (RH) and black globe temperature (BGT), inside and outside of facilities, were measured hourly by a period of 24:00 h. Respiratory frequency (RF), rectal temperature (RT) and skin temperature (ST) were taken at 1:00 p.m., 2:00 p.m, 3:00 p.m. and 4:00 p.m. in all animals submitted to the treatments. The environmental variables, DBT $\left(31.5^{\circ} \mathrm{C}\right)$ and $\mathrm{BGT}\left(32.5^{\circ} \mathrm{C}\right)$, and thermal comfort indexes, THI (79.0) and BGHI (81.0), were higher with 12 min of sprinkling frequency than the other treatments. The RH was higher with $14 \min (60.3 \%)$ than with $12 \min (51.0 \%)$. It was verified that in relation to physiological parameters, dairy cows with $12 \mathrm{~min}$ of sprinkling frequency had higher rectal temperature $\left(39.1^{\circ} \mathrm{C}\right)$ and respiratory frequency $\left(76.0 \mathrm{mov} \mathrm{min}^{-1}\right)$ when compared to the treatments of 14 and $16 \mathrm{~min}$. To avoid unnecessary water waste in the system should be used the interval of $16 \mathrm{~min}$ resulting in the smallest water amount used by the sprinkling cooling system.

KEYWORDS: environmental, dairy cows, heat stress.

\footnotetext{
${ }^{1}$ Extraído da tese apresentada pelo primeiro autor à ESALQ/USP. Trabalho financiado pela FAPESP.

${ }^{2}$ Zootecnista, Doutor, Centro APTA Bovinos de Leite (IZ), Nova Odessa - SP, Fone: (0XX19) 3466.9435, matarazzo@iz.sp.gov.br

${ }^{3}$ Eng ${ }^{0}$ Agrícola, Prof. Doutor, Departamento de Engenharia Rural, ESALQ/USP, Piracicaba - SP.

${ }^{4}$ Eng ${ }^{\mathrm{O}}$ Agrônomo, Mestre, Departamento de Engenharia Rural, ESALQ/USP, Piracicaba - SP.

${ }^{5}$ Zootecnista, Prof. Adjunto, Departamento de Tecnologia Rural e Animal, UESB, Itapetinga - BA.

Recebido pelo Conselho Editorial em: 17-2-2006
}

Aprovado pelo Conselho Editorial em: 12-7-2006 


\section{INTRODUÇÃO}

A produção de leite em ambiente tropical pode ser melhorada com a adoção de tecnologias que possibilitem o manejo estratégico do rebanho. A movimentação e o resfriamento evaporativo do ar, o uso de sombras para minimizar o efeito da radiação solar são artifícios empregados para potencializar a dissipação de calor (WEST, 2003).

O sistema de resfriamento adiabático evaporativo (SRAE) expandiu-se rapidamente em climas quentes, devido à sua simplicidade técnica, praticidade e relação custo/benefício favorável, resultando em aceitação por muitos produtores (LIN et al., 1998), podendo ser obtido por vários processos, entre os quais se destacam: a nebulização, a microaspersão e a aspersão sobre os animais ou telhados.

A aspersão não tem por finalidade resfriar o ar, pois emprega gotas com tamanho maior, de modo a promover o umedecimento dos pêlos das vacas. $\mathrm{O}$ animal se resfria com a evaporação da água, por meio da pele e pêlos, permitindo a troca de calor mais eficiente, quando comparada apenas com a sudação. Quando associada à ventilação natural ou forçada, aumenta a eficiência do resfriamento, por acelerar o processo de evaporação (BUCKLIN \& BRAY, 1998).

Para regiões com umidade relativa elevada, os sistemas em que os aspersores funcionam de maneira intermitente, ou seja, são acionados durante determinado intervalo e então desligados, enquanto os ventiladores movimentam grandes volumes de ar ao redor dos animais, tem se mostrado bastante eficiente (TURNER, 2001).

As recomendações iniciais para a intermitência de acionamento do SRAE sugeriram que o intervalo deveria ser de $15 \mathrm{~min}$ (BROUK et al., 2001). Entretanto, estudos realizados posteriormente revelaram que estratégias distintas de intermitência deveriam ser adotadas para diferentes níveis de estresse térmico. HILLMAN et al. (2001) avaliaram diferentes intervalos de acionamento para a aspersão: sem molhar e molhando a cada 20 ou $40 \mathrm{~min}$. A água era aplicada durante 1,5 min até que o excesso escorresse pelo flanco da vaca e as intermitências eram associadas a diferentes fluxos de ar: desligado, 0,$1 ; 0,9$ e 2,2 $\mathrm{m} \mathrm{s}^{-1}$. A temperatura da pele caiu rapidamente quando a intermitência foi 20 min e fluxo de ar igual a $2,2 \mathrm{~m} \mathrm{~s}^{-1}$.

Cabe ainda ressaltar que o SRAE por aspersão utiliza grandes volumes de água. Portanto, deveria ser empregado o intervalo com a menor taxa de aplicação de água, a fim de evitar desperdícios e preservar esse recurso natural.

Dessa forma, o presente trabalho teve como objetivo avaliar o período de intermitência de acionamento do SRAE por aspersão na linha de alimentação, em instalação do tipo freestall, usando os índices de conforto térmico (ITU e ITGU) e as respostas fisiológicas de vacas em lactação.

\section{MATERIAL E MÉTODOS}

Foram utilizadas 12 vacas holandesas, multíparas, na fase intermediária de lactação, com peso médio de $600 \mathrm{~kg}$ e produção média de $30 \mathrm{~kg}$ de leite por dia. O trabalho foi conduzido com rebanho comercial, localizado no município de São Pedro - SP. O clima da região, segundo a classificação Köeppen é do tipo Cwa , ou seja, quente e úmido com estação chuvosa no verão e seco no inverno.

Os tratamentos foram aplicados de maneira aleatória em três períodos distintos, de modo que cada tratamento foi avaliado para o período em questão:

- Tratamento 1: intermitência de 12 min ( 1 min 25 s ligado e 10 min 35 s desligado);

- Tratamento 2: intermitência de 14 min (1 min 25 s ligado e 12 min 35 s desligado);

- Tratamento 3: intermitência de 16 min (1 min 25 s ligado e 14 min 35 s desligado). 
O sistema de aspersão, em tubo de PVC, foi montado 2,5 $\mathrm{m}$ acima da linha de alimentação, com espaçamento entre bicos de $1 \mathrm{~m}$. A vazão empregada foi a mesma em todos os tratamentos, ou seja, $0,71 \mathrm{~L}$ de água foram aspergidos no ambiente durante o intervalo de $1 \mathrm{~min} 25 \mathrm{~s}$. No período de uma hora, para o tratamento de $12 \mathrm{~min}$, o sistema foi acionado cinco vezes, resultando no consumo de $3,6 \mathrm{~L} \mathrm{~h}^{-1}$. Para a intermitência de $14 \mathrm{~min}$, acionou-se 4,3 vezes e para o intervalo de 16 minutos, 3,8

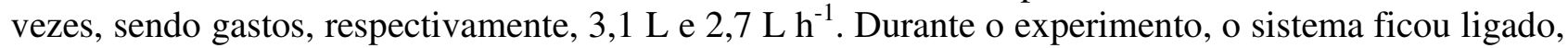
em média, nove horas por dia. Dessa forma, tem-se o consumo médio de água de 32,0 L, 27,9 L e $24,3 \mathrm{~L} \mathrm{vaca}^{-1} \mathrm{dia}^{-1}$, respectivamente, para os tratamentos de $12 \mathrm{~min}, 14 \mathrm{~min}$ e $16 \mathrm{~min}$.

Foram utilizados ventiladores com diâmetro de $0,9 \mathrm{~m}$, espaçados a cada $11 \mathrm{~m}$, equipados com motor de $1 / 4 \mathrm{cv}$, vazão de $300 \mathrm{~m}^{3} \mathrm{~h}^{-1}, 495 \mathrm{rpm}$, com capacidade de produzir movimentação de ar de até $2,5 \mathrm{~m} \mathrm{~s}^{-1}$ na altura do dorso do animal.

A coleta de dados teve a duração de 116 dias, nos meses de janeiro a maio de 2003. Entretanto, o período foi caracterizado pela ocorrência de intensa precipitação, o que limitou a utilização do SRAE. Sendo assim, foram selecionados para a análise estatística dos dados 15 dias críticos, cinco dias para cada tratamento. A seleção desses dias baseou-se no conceito de entalpia crítica ( $\left.\mathrm{kJ} \mathrm{kg} \mathrm{ar} \mathrm{seco}{ }^{-1}\right)$, a qual foi atingida com TBS igual ou superior a $24{ }^{\circ} \mathrm{C}$ e UR igual a $76 \%$. Para as condições do presente trabalho, esse valor foi estimado em $63,0 \mathrm{~kJ} \mathrm{~kg}$ ar $\mathrm{seco}^{-1}$, o qual foi calculado pelo programa computacional PsyCalc $98^{\circledR}$, considerando a pressão barométrica local igual a $708 \mathrm{~mm} \mathrm{Hg}$ (JOHNSON \& VANJONACK, 1976). Portanto, durante o período experimental, os dias que apresentaram entalpia igual ou superior a $63,0 \mathrm{~kJ} \mathrm{~kg}$ ar seco $^{-1}$ no ambiente externo à instalação, foram selecionados para a análise. Os valores das variáveis meteorológicas registradas no ambiente externo foram posteriormente incorporados ao modelo estatístico, de modo a realizar o ajuste adequado (covariável) para a interpretação dos dados.

As variáveis meteorológicas registradas no interior da instalação e no ambiente externo foram temperatura do bulbo seco (TBS), umidade relativa do ar (UR\%) e temperatura do globo negro (TGN). A partir dos valores encontrados para TBS, UR e TGN, calcularam-se o índice de temperatura e umidade (ITU) proposto por THOM (1959) e o índice de temperatura de globo negro e umidade (ITGU), de acordo com BUFFINGTON et al. (1981). As leituras foram realizadas em intervalos horários por sensores acoplados a um sistema eletrônico de aquisição de dados $\left(\mathrm{HOBO}^{\circledR} \mathrm{H} 8\right)$ ao longo das $24 \mathrm{~h}$, durante todo o período experimental.

A coleta dos dados fisiológicos (temperatura retal (TR), temperatura do pelame (TP) e freqüência respiratória (FR)) foram realizadas ao longo do período experimental às $13 ; 14 ; 15$ e $16 \mathrm{~h}$, em todos os animais submetidos aos tratamentos. Da mesma forma, selecionaram-se para a análise dos dados fisiológicos as respostas obtidas nos mesmos 15 dias críticos (cinco em cada tratamento), considerados para as variáveis ambientais.

As análises estatísticas foram realizadas com o procedimento para modelos mistos (Proc Mixed) do SAS (1998), admitindo-se modelo com medidas repetidas no tempo. Para as variáveis ambientais, adotou-se o delineamento experimental inteiramente casualizado, com os dias sendo usados como repetições. A fim de ajustar as respostas médias dos tratamentos, já que os períodos de observação não foram simultâneos, incorporou-se ao modelo uma co-variável. Dessa forma, a análise da TBS teve como variável auxiliar a sua correspondente registrada no ambiente externo, e assim sucessivamente, para UR, TGN, ITU, ITGU e entalpia. Os dados fisiológicos foram arranjados conforme o delineamento experimental inteiramente casualizado, com os animais sendo usados como repetições. 


\section{RESULTADOS E DISCUSSÃO}

Na Tabela 1, apresentam-se os valores médios das variáveis meteorológicas e índices de conforto térmico registrados nos tratamentos avaliados, no intervalo das 13 às $16 \mathrm{~h}$, durante os dias críticos selecionados para análise.

TABELA 1. Valores médios e erros-padrão da média das variáveis meteorológicas e índices de conforto térmico registrados no intervalo das 13 às $16 \mathrm{~h}$.

\begin{tabular}{lccc}
\hline Variáveis Meteorológicas & \multicolumn{3}{c}{ Tratamentos } \\
\cline { 2 - 4 } & $12 \mathrm{~min}$ & $14 \mathrm{~min}$ & $16 \mathrm{~min}$ \\
\hline Temperatura de bulbo seco, ${ }^{\circ} \mathrm{C}$ & $31,5 \pm 0,55 \mathrm{a}$ & $28,8 \pm 0,50 \mathrm{~b}$ & $28,9 \pm 0,54 \mathrm{~b}$ \\
Umidade relativa, \% & $51,0 \pm 1,80 \mathrm{a}$ & $60,3 \pm 1,90 \mathrm{~b}$ & $54,6 \pm 1,70 \mathrm{ab}$ \\
Temperatura de globo negro, ${ }^{\circ} \mathrm{C}$ & $32,5 \pm 0,60 \mathrm{a}$ & $27,5 \pm 0,59 \mathrm{~b}$ & $29,2 \pm 0,66 \mathrm{~b}$ \\
Índice de temperatura e umidade & $79,0 \pm 0,46 \mathrm{a}$ & $78,0 \pm 0,38 \mathrm{~b}$ & $77,0 \pm 0,45 \mathrm{~b}$ \\
Índice de globo negro e umidade & $81,0 \pm 0,52 \mathrm{a}$ & $76,0 \pm 0,59 \mathrm{~b}$ & $77,0 \pm 0,51 \mathrm{~b}$ \\
Entalpia, kJ kg de ar seco ${ }^{-1}$ & $70,5 \pm 1,43 \mathrm{a}$ & $71,1 \pm 1,42 \mathrm{a}$ & $67,0 \pm 1,68 \mathrm{a}$ \\
\hline
\end{tabular}

a, b - médias seguidas por letras minúsculas distintas, nas linhas, diferem entre si, pelo teste t de Student $(\mathrm{P}<0,05)$.

Foram constatadas diferenças $(\mathrm{P}<0,05)$ entre os tratamentos para a TBS. Essa variável mostrouse mais elevada no tratamento de $12 \mathrm{~min}\left(31,5^{\circ} \mathrm{C}\right)$ quando comparada com os tratamentos de 14 min $\left(28,8^{\circ} \mathrm{C}\right)$ e 16 min $\left(28,9^{\circ} \mathrm{C}\right)$. Os tratamentos de 14 e 16 min não diferiram entre si. A adoção dos diferentes intervalos de acionamento do SRAE não reduziu a TBS para os níveis de conforto térmico. Por outro lado, os resultados obtidos estão de acordo com BUCKLIN \& BRAY (1998), pois o sistema de aspersão não tem a finalidade de resfriar o ar, e sim de usar gotas largas de água para molhar o pêlo e a pele, melhorando a sensação térmica das vacas em lactação.

A variável UR mostrou-se mais elevada $(\mathrm{P}<0,05)$ no tratamento de $14 \min (60,3 \%)$ quando comparada ao tratamento de $12 \mathrm{~min}(51,0 \%)$. Possivelmente, essa variação ocorreu devido à evaporação mais rápida da água no tratamento de $12 \mathrm{~min}$. De maneira geral, os intervalos de intermitência estudados não resultaram em umidade excessiva, ou seja, maior que $70 \%$ no interior da instalação. Temperatura ambiente e umidade relativa elevada poderiam limitar o desempenho das vacas confinadas, pois diminuem sua habilidade em dissipar calor. Isso ocorre porque, em condições de umidade elevada, o ar saturado irá inibir a evaporação da água pela pele e sistema respiratório, proporcionando ambiente ainda mais estressante para o animal (SOTA, 1996).

Foram observadas diferenças $(\mathrm{P}<0,05)$ entre os tratamentos para a TGN. O tratamento de $12 \mathrm{~min}$ $\left(32,5^{\circ} \mathrm{C}\right)$ apresentou TGN mais elevada quando comparado aos tratamentos de $14 \min \left(27,5^{\circ} \mathrm{C}\right)$ e 16 min $\left(29,2^{\circ} \mathrm{C}\right)$, sendo que esses últimos não diferiram entre si. Os valores de TGN apresentaram o mesmo comportamento verificado para a TBS. Os menores valores de TGN obtidos para os tratamentos de 14 min e 16 min podem estar associados ao efeito convectivo promovido pela presença de ventilação. O termômetro de globo negro fornece, em uma só medida, a indicação dos efeitos combinados da temperatura do ar, temperatura radiante e velocidade do vento (BOND \& KELLY, 1955). Constatou-se, mais uma vez, que as condições do ambiente foram superiores à zona de termoneutralidade dos animais.

O ITU foi mais elevado $(\mathrm{P}<0,05)$ no tratamento de 12 min $(79,0)$ comparado ao tratamento de $14 \min (78,0)$ e de 16 min $(77,0)$, sendo que esses últimos não diferiram entre si. Existem vários relatos na literatura sobre os limites do ITU (JOHNSON et al., 1963; HAHN \& MADER, 1997). Trabalhos realizados por HAHN (1985) apontaram que o THI igual ou menor que 70 expressa condição normal; valor entre 71 e 78 é crítico; entre 79 e 83 a situação é de perigo e, acima de 83 , situação de emergência está presente. Os valores do ITU aqui verificados para os tratamentos de 14 e 
16 min permaneceram na faixa considerada crítica; já a situação de perigo encontrada para a intermitência de 12 min deve-se, possivelmente, à TBS elevada registrada nesse tratamento.

De modo semelhante, foram observadas diferenças $(\mathrm{P}<0,05)$ entre os tratamentos para o ITGU. Tal índice apresentou-se mais elevado no tratamento de 12 min $(81,0)$, quando comparado ao tratamento de 14 min $(76,0)$ e de 16 min $(77,0)$, sendo que esses últimos não diferiram entre si. $\mathrm{O}$ ITGU apresentou o mesmo comportamento verificado para a TGN; assim, o maior valor determinado para a intermitência de 12 min estaria associado à TGN elevada registrada nesse tratamento. O ITGU foi reduzido em 4,4 e 3,5 unidades em relação à intermitência de $12 \mathrm{~min}$, respectivamente, quando empregados os intervalos de 14 e 16 min. De acordo com BAÊTA \& SOUZA (1997), valores de ITGU até 74 definem condição de conforto para os bovinos, entre 75 e 78 a situação é de alerta, de 79 a 84 caracterizam perigo e, acima desse, depara-se com situação de emergência. Sendo assim, nas condições presentes, os valores de ITGU encontrados permaneceram nas faixa de alerta para os tratamentos de 14 e de 16 min e situação de perigo para a intermitência de $12 \mathrm{~min}$.

A quantidade de energia presente na massa de ar seco, que é expressa pela variável entalpia, não diferiu $(\mathrm{P}>0,05)$ entre os tratamentos. Os valores médios encontrados foram 70,5; 71,1 e 67,9 kJ kg de $\operatorname{ar} \operatorname{seco}^{-1}$, respectivamente, para os tratamentos de $12 ; 14$ e 16 min. Nas condições do experimento, a entalpia na faixa de termoneutralidade corresponderia a $63,0 \mathrm{~kJ} \mathrm{~kg} \mathrm{de} \mathrm{ar} \mathrm{seco-1;} \mathrm{verificou-se,} \mathrm{portanto,}$ que os resultados obtidos foram superiores aos sugeridos pela literatura. Nessas situações, os tratamentos adotados não foram eficientes a ponto de promover mudanças na quantidade de calor no interior da instalação.

O consumo médio de água pelo SRAE foi 32,0; 27,9 e 24,3 $\mathrm{L} \mathrm{vaca}^{-1} \mathrm{dia}^{-1}$, respectivamente, para os tratamentos de $12 \mathrm{~min}, 14 \mathrm{~min}$ e $16 \mathrm{~min}$, valores consideravelmente inferiores aos dados disponíveis na literatura. O SRAE, geralmente, resulta em grandes volumes de águas residuais, por isso sugere-se empregar a menor taxa de aplicação, a fim de evitar desperdícios e preservar esse recurso natural.

MEANS et al. (1992) avaliaram três taxas de água (313,4; 492,9 e 704,1 $\mathrm{L} \mathrm{hora}^{-1}$ ) no sistema de aspersão em freestall para vacas em lactação. Os animais eram aspergidos durante 1,5 min em cada ciclo de $15 \mathrm{~min}$, quando a temperatura interna do freestall era superior a $25,6^{\circ} \mathrm{C}$. Os resultados indicaram que a produtividade, a temperatura retal e a taxa respiratória não foram alteradas com a aplicação dos tratamentos.

Na Tabela 2, são apresentados os valores médios e erros-padrão da média verificados para a temperatura retal (TR), frequiência respiratória (FR) e temperatura do pelame (TP) durante o período analisado.

Os valores médios encontrados para a temperatura retal foram $39,1^{\circ} \mathrm{C}, 38,8^{\circ} \mathrm{C}$ e $38,7{ }^{\circ} \mathrm{C}$, respectivamente, para os tratamentos de 12; 14 e $16 \mathrm{~min}$. A análise dos dados identificou a presença de interação entre tratamento e hora $(\mathrm{P}<0,05)$ para a TR. $\mathrm{O}$ desdobramento da interação mostrou-se significativo para os horários das 14; 15 e $16 \mathrm{~h}$. Quando os animais foram submetidos ao tratamento de $12 \mathrm{~min}$, os valores de TR foram maiores $\left(39,1^{\circ} \mathrm{C}\right)$ do que quando aplicados os tratamentos de $14 \mathrm{~min}$ $\left(38,7^{\circ} \mathrm{C}\right)$ e de $16 \min \left(38,7^{\circ} \mathrm{C}\right)$; esse efeito foi verificado para o horário das $14 \mathrm{~h}$ e $15 \mathrm{~h}$. Por outro lado, às $16 \mathrm{~h}$, os animais mantidos no tratamento de 12 min apresentaram maior TR $\left(39,1^{\circ} \mathrm{C}\right)$, comparados com a aplicação do intervalo de $16 \min \left(38,7^{\circ} \mathrm{C}\right)$. Entretanto, os valores verificados para a TR permaneceram na faixa de normalidade para bovinos, que está entre 38 e $39,5^{\circ} \mathrm{C}$ (DU PREEZ, 2000). 
TABELA 2. Valores médios e erros-padrão da média para as variáveis fisiológicas das vacas em lactação medidas das 13 às $16 \mathrm{~h}$.

\begin{tabular}{lccccc}
\hline TR & $13 \mathrm{~h}$ & $14 \mathrm{~h}$ & $15 \mathrm{~h}$ & $16 \mathrm{~h}$ & Efeito \\
\hline $12 \mathrm{~min}$ & $39,0 \pm 0,11 \mathrm{a}$ & $39,1 \pm 0,11 \mathrm{a}$ & $39,3 \pm 0,11 \mathrm{a}$ & $39,1 \pm 0,11 \mathrm{a}$ & Cúbico \\
$14 \mathrm{~min}$ & $38,8 \pm 0,11 \mathrm{a}$ & $38,7 \pm 0,11 \mathrm{~b}$ & $38,8 \pm 0,11 \mathrm{~b}$ & $38,8 \pm 0,11 \mathrm{ab}$ & NS \\
$16 \mathrm{~min}$ & $38,8 \pm 0,11 \mathrm{a}$ & $38,7 \pm 0,11 \mathrm{~b}$ & $38,7 \pm 0,11 \mathrm{~b}$ & $38,7 \pm 0,11 \mathrm{~b}$ & Linear $^{2}$ \\
\hline FR & & & & \\
\hline $12 \mathrm{~min}$ & $80,0 \pm 0,68$ & $76,0 \pm 0,68$ & $76,0 \pm 0,68$ & $68,0 \pm 0,11$ & Linear $^{3}$ \\
$14 \mathrm{~min}$ & $60,0 \pm 0,68$ & $60,0 \pm 0,68$ & $60,0 \pm 0,68$ & $60,0 \pm 0,11$ & Linear $^{3}$ \\
16 min & $64,0 \pm 0,68$ & $60,0 \pm 0,68$ & $60,0 \pm 0,68$ & $56,0 \pm 0,11$ & Linear $^{3}$ \\
\hline TP & & & & & \\
12 min & $31,7 \pm 0,22 \mathrm{a}$ & $31,7 \pm 0,22 \mathrm{a}$ & $31,5 \pm 0,22 \mathrm{a}$ & $31,3 \pm 0,22 \mathrm{a}$ & Linear $^{4}$ \\
$14 \min$ & $31,2 \pm 0,22 \mathrm{ab}$ & $31,9 \pm 0,22 \mathrm{a}$ & $30,9 \pm 0,22 \mathrm{c}$ & $31,3 \pm 0,22 \mathrm{a}$ & Cúbico $^{5}$ \\
16 min & $30,7 \pm 0,22 \mathrm{~b}$ & $30,7 \pm 0,22 \mathrm{~b}$ & $30,2 \pm 0,22 \mathrm{~b}$ & $28,9 \pm 0,22 \mathrm{~b}$ & Linear $^{6}$ \\
\hline
\end{tabular}

$\mathrm{a}, \mathrm{b}$ - Médias seguidas por letras minúsculas distintas, nas colunas, diferem pelo Teste $\mathrm{t}$ de Student $(\mathrm{P}<0,05)$

${ }^{1} \mathrm{Y}=-0,08 \mathrm{x}^{3}+3,5 \mathrm{x}^{2}-50,2 \mathrm{x}+274,3\left(\mathrm{R}^{2}=0,99\right) ;{ }^{2} \mathrm{Y}=-0,03 \mathrm{x}+39,2\left(\mathrm{R}^{2}=0,60\right) ;{ }^{3} \mathrm{Y}=-2,4 \mathrm{x}+98,8\left(\mathrm{R}^{2}=0,90\right)$

${ }^{4} \mathrm{Y}=-0,14 \mathrm{x}+33,6\left(\mathrm{R}^{2}=0,89\right) ;{ }^{5} \mathrm{Y}=0,35 \mathrm{x}^{3}-15,1 \mathrm{x}^{2}+214,6 \mathrm{x}-983,1\left(\mathrm{R}^{2}=0,99\right) ;{ }^{6} \mathrm{Y}=-0,32 \mathrm{x}+35,0\left(\mathrm{R}^{2}=0,90\right)$.

Os resultados observados entre os horários de avaliação revelaram efeito cúbico para o tratamento de $12 \mathrm{~min}$. Nessa condição, a TR apresentou valores mais elevados a partir das $13 \mathrm{~h}$. Esses dados corroboram o trabalho de BACCARI JÚNIOR et al. (1979), que, na faixa de termoneutralidade, verificaram para bovinos holandeses correlação mais alta $(r=0,96)$ da TR com a hora do dia do que com a temperatura do ar $(\mathrm{r}=0,71)$. A partir das $15 \mathrm{~h}$, a temperatura do ar começou a cair, enquanto a temperatura retal continuou a aumentar, mostrando que, durante o dia, essa se eleva em função do metabolismo animal que propriamente da temperatura ambiente. Por outro lado, a TR das vacas mantidas no tratamento de 16 min apresentou efeito linear, sendo que às $13 \mathrm{~h}$ se mostrou mais elevada $\left(38,8^{\circ} \mathrm{C}\right)$, e a partir daí se manteve invariável $\left(38,7^{\circ} \mathrm{C}\right)$ ao longo do dia.

A duração da aspersão é uma variável crítica para garantir a efetividade do sistema de resfriamento. Molhar a vaca a cada 15; 30 e 45 min reduziu a temperatura retal, respectivamente, em 0,$6 ; 0,7$ e $1,0^{\circ} \mathrm{C}$. Assim, o período de tempo em que o sistema fica ligado para promover o umedecimento do pêlo do animal, bem como a taxa de ventilação, teve efeitos dramáticos no resfriamento corporal encontrado (FLAMENBAUM et al., 1986).

A frequiência respiratória diferiu $(\mathrm{P}<0,05)$ entre os tratamentos avaliados. A FR dos animais, no tratamento de $12 \mathrm{~min}$, foi maior $\left(76 \mathrm{mov} \mathrm{min}^{-1}\right)$ do que quando foram submetidos à intermitência de 14 $\min \left(60 \mathrm{mov} \mathrm{min}^{-1}\right)$ ou $16 \mathrm{~min}\left(60 \mathrm{mov} \mathrm{min}^{-1}\right)$. Os resultados observados entre os horários de avaliação revelaram efeito linear para todos os tratamentos, sendo que a FR diminuiu ao longo do dia. A FR de 60 mov min $^{-1}$ indica animais com ausência de estresse térmico ou esse é mínimo (HAHN \& MADER 1997). Nas condições do presente experimento, os valores de FR encontrados, nos tratamentos de 14 e $16 \mathrm{~min}$, foram iguais a esses; por outro lado, para a intermitência de $12 \mathrm{~min}$, a FR mostrou-se mais elevada (76 mov $\mathrm{min}^{-1}$ ). De acordo com BACCARI JR. et al. (1995), sob temperatura de $31^{\circ} \mathrm{C}$ e THI igual a 79, as vacas holandesas apresentaram freqüência respiratória de $68 \mathrm{mov} \mathrm{min}^{-1}$, com amplitude de variação de 44 a 108.

Pesquisas conduzidas por FRAZZI et al. (1998) concluíram que a utilização de sistemas de condicionamento ambiental em instalações para vacas leiteiras proporcionou a diminuição da temperatura retal, a redução da taxa respiratória, melhorou o rendimento produtivo e as características do leite das horas mais quentes do dia, se comparadas com vacas em instalações que possuem apenas ventilação natural. 
Os valores médios encontrados para a temperatura do pelame foram $31,6{ }^{\circ} \mathrm{C}, 31,3{ }^{\circ} \mathrm{C}$ e $30,4{ }^{\circ} \mathrm{C}$, respectivamente, para os tratamentos de 12;14 e $16 \mathrm{~min}$. A análise dos dados identificou a presença de interação entre tratamento e horário de avaliação $(\mathrm{P}<0,05)$ para a TP. $\mathrm{O}$ desdobramento da interação mostrou-se significativo para todos os horários avaliados. Em todos esses instantes, as vacas submetidas à intermitência de 12 min apresentaram maiores valores para a TP, comparadas com a aplicação do tratamento de $16 \mathrm{~min}$. Ainda, às $15 \mathrm{~h}$, a TP dos animais do tratamento de 12 min foi maior que as do tratamento de $14 \mathrm{~min}$. Quando aplicado o tratamento de $14 \mathrm{~min}$, verificaram-se, às $14 \mathrm{~h}\left(31,9^{\circ} \mathrm{C}\right)$, às $15 \mathrm{~h}\left(30,9^{\circ} \mathrm{C}\right)$ e às $16 \mathrm{~h}\left(31,3^{\circ} \mathrm{C}\right)$, maiores valores de $\mathrm{TP}$, comparado ao tratamento de 16 min. Os resultados observados entre os horários de avaliação revelaram efeito linear para os tratamentos de 12 e 16 min, sendo que a TP diminuiu ao longo do dia. O efeito cúbico foi verificado para a intermitência de $14 \mathrm{~min}$, uma vez que a TP oscilou durante o dia, apresentando-se mais elevada às 14 e às $15 \mathrm{~h}$, com ligeiro decréscimo a partir das $15 \mathrm{~h}$.

A aspersão de água sobre o animal resfria imediatamente a superfície do corpo em até $4{ }^{\circ} \mathrm{C}$ e reduz a taxa respiratória a 18 movimentos por min. A perda de calor por evaporação é favorecida quando a pele está molhada, representando $82 \%$ da perda total. CHASTAIN \& TURNER (1994) estimaram como 0,9 L o volume de água necessário para saturar o pêlo do dorso de uma vaca holandesa de $600 \mathrm{~kg}$. Os bicos deveriam ter taxa de 0,45 a 1,9 $\mathrm{L} \mathrm{min}^{-1}$, e o tempo de aspersão de água, entre 0,5 a 2 min.

A quantidade de água adotada no presente experimento foi $0,71 \mathrm{~L}$ durante o intervalo de $1 \mathrm{~min}$ $25 \mathrm{~s}$. O tipo de bico aspersor empregado no SRAE permitiu que o pêlo e a pele da região dorsal do animal fossem saturados nesse intervalo, evitando, assim, gastos de água desnecessários. Em média, a TP foi reduzida em $1,05^{\circ} \mathrm{C}$ quando utilizada a intermitência de 16 min em relação aos demais intervalos.

Os efeitos do sistema de resfriamento adiabático por aspersão associado à ausência ou presença de fluxo de ar (ventilação forçada) e seus reflexos sobre a temperatura da pele foram avaliados por BROUK et al. (2001). Os resultados apontaram que os tratamentos associados à ventilação foram responsáveis por menores temperaturas de pele. É importante ressaltar que os efeitos evaporativos e convectivos na perda de calor não são facilmente separáveis. Assim, a velocidade do ar é um fator importante que influencia no processo de transferência evaporativa (KADZERE et al., 2002).

\section{CONCLUSÕES}

As intermitências de 14 e 16 min proporcionaram condições de conforto térmico (variáveis meteorológicas) semelhantes para as vacas em lactação. Entretanto, o intervalo de 16 min empregou menor quantidade de água, podendo ser adotado a fim de evitar desperdícios desnecessários pelo sistema de resfriamento adiabático evaporativo. As intermitências de 14 e 16 min proporcionaram menores valores para as variáveis termorregulatórias de temperatura retal, frequência respiratória e temperatura do pelame, mostrando-se eficiente na redução do estresse térmico de vacas em lactação.

\section{REFERÊNCIAS}

BACCARI JUNIOR, F.; CAMPOS NETO, O.; ROCHA, G.P. Variação fisiológica da temperatura retal das 8 às $18 \mathrm{~h}$ em bovinos holandeses. Correlação com a temperatura ambiente e hora do dia. In: JORNADA CIENTÍFICA DA ASSOCIAÇÃO DOS DOCENTES DO CÂMPUS DE BOTUCATU, 8., 1979, Botucatu. Anais... Botucatu: FMVZ, 1979. p.149-53.

BACCARI JUNIOR, F.; AGUIAR, I.S.; TEODORO, S.M. Hipertemia, taquipinéia e taquicardia em vacas holandesas malhadas de vermelho sob estresse térmico. In: CONGRESSO BRASILEIRO DE BIOMETEOROLOGIA, 3., 1995, Jaboticabal. Anais... Jaboticabal: FUNEP, 1995. p.15-16. 
BAÊTA, F.C.; SOUZA, C.F. Ambiência em edificações rurais - conforto animal. Viçosa: Universidade Federal de Viçosa, 1997. 246 p.

BOND, T. E.; KELLY, C. F. The globe thermometer in agriculture research. Agriculture Engineering, Columbia, v.36, p.251-60, 1955.

BROUK, M.J.; SMITH, J.F.; HARNER III, J.P. Efficiency of modified evaporative cooling in Midwest dairy freestall barn In: INTERNATIONAL LIVESTOCK ENVIRONMET SYMPOSIUM, 6., 2001, Louisville. Proceedings... Louisville: ASAE, 2001a.1 CD-ROM.

BUCKLIN, R.A.; BRAY, D.R. The American experience in dairy management warm and hot climates. In: SIMPÓSIO BRASILEIRO DE AMBIÊNCIA NA PRODUÇÃO DE LEITE, 1., 1998, Piracicaba. Anais... Piracicaba: FEALQ, 1998. p.156-74.

BUFFINGTON, D.E.; COLLAZO-AROCHO, A.; CANTON, G.H. Black globe-humidity index (BGHI) as comfort equation for dairy cows. Transactions of ASAE, St. Joseph, v.24, n.3, p.711-14, 1981.

CHASTAIN, J.P.; TURNER, L.W. Practical results of a model of direct evaporative cooling of dairy cows. In: INTERNATIONAL DAIRY HOUSING CONFERENCE, 3., 1994. Orlando. Proceedings... Orlando: ASAE, 1994. p.337-52.

DU PREEZ, J.H. Parameters for the determination and evaluation of heat stress in dairy cattle in South Africa. Onderstepoort Journal Veterinary Research, Pretoria, v.67, n.3, p.263-71, 2000.

FLAMENBAUM, I.; WOLFENSON, D.; MANEN, M. Cooling dairy cattle by a combination of sprinkling and forced ventilation and its implementation in the shelter system. Journal of Dairy Science, Champaign, v.69, n.12, p.3140-7, 1986.

FRAZZI, E.; CALAMARI, L.; CALEGARI, F. Behavior of dairy cows in response to different barn cooling systems. In: INTERNATIONAL DAIRY HOUSING CONFERENCE, 4., 1998, St. Louis. Proceedings... St. Louis: ASAE, 1998. p.387-94.

HAHN, G.L. Management and housing of farm animals in hot environment. In: YOUSEF, M.K. Stress physiology in livestock. Boca Raton: CRC Press, 1985. v.2, p.151-74.

HAHN, G.L.; MADER, T.L. Heat waves in relation o thermoregulation, feeding behavior, and mortality of feedlot cattle. In: INTERNATIONAL LIVESTOCK ENVIRONMENT SYMPOSIUM, 5., 1997, Minnesota. Proceedings... St. Joseph: ASAE, 1997. p.125-9.

HILLMAN, P.E.; GEBREMEDHIN, K.G.; PARKHRST, A. Evaporative and convective cooling of cows in a hot and humid environment. In: INTERNATIONAL LIVESTOCK ENVIRONMET SYMPOSIUM, 6., 2001, Louisville. Proceedings... Louisville: ASAE, 2001. 1 CD-ROM.

JOHNSON, H.D.; RAGSDALE, A.C.; BERRY, I. L. Temperature-humidity effects including influence of acclimation in feed and water consumption of Holstein cattle. Columbia: Missouri Agricultural Experimental Station, 1963 (Research Bulletin, 846).

JOHNSON, H.D.; VANJONACK, W.J. Effects of environmental and other stressors on blood hormone patterns in lactating animals. Journal of Dairy Science, Champaign, v.59, n.9, p.1603-17, 1976.

KADZERE, C.T.; MURPHY, M.R.; SILANIKOVE, N.; MALTZ, E. Heat stress in lactating dairy cows: a review. Livestock Production Science, Amsterdam, v.77, n.1, p.59-91, 2002.

LIN, J.C.; MOSS, B.R.; KOON, J.L.; FLOOD, C.A. Comparison of various fan, sprinkler, and mist systems in reducing heat stress in dairy cows. Applied Engineering in Agriculture, St. Joseph, v.14, n.2, p.177-82, 1998. 
MEANS, S.L.; BUCKLIN, R.A.; NORDSTEDT, R.A. Water application rates for a sprinkler and fan dairy cooling system in hot, humid climates. Applied Engineering in Agriculture, St. Joseph, v.8, n.3, p.375-9, 1992.

PSYCALC 98. Psychrometric calculator. Bradford: Linric Company, 1997.

SAS INSTITUTE. User'guide: statistics. SAS (Release 6.03). Cary, 1998. 620 p.

SOTA, R. L. Fisilogia ambiental: mecanismos de respuestas del animal al estress calórico. In: JORNADA DE MANEJO DEL ESTRESS CALÓRICO, 1., 1996, La Plata. Anais... La Plata: EDULP, 1996. p.1-43.

THOM, E.C. The discomfort index. Weatherwise, Washington, v.12, n.2, p.57-9, 1959.

TURNER, L.W. Global position systems (GPS) and grazing behavior in cattle. In: INTERNATIONAL LIVESTOCK ENVIRONMENT SYMPOSIUM, 6., 2001, Louisville. Proceedings... Louisville: ASAE, 2001. 1 CD-ROM.

WEST, J.W. Effects of heat-stress on production in dairy cattle. Journal of Dairy Science, Champaign, v.86, n.6, p.2131-44, 2003. 